\title{
Testosterona, Colesterol, ADH e a Agressividade em Touros de Lide sob Stress
}

\author{
Gouveia, A.J.
}

Instituto Nacional de Investigação Agrária e Veterinária. Oeiras. Portugal.

\section{PalaVRas chaVe ADICIONAIS}

Hormonas.

Stress.

Vasopressina.

Comportamento.

Tourada.

\section{RESUMO}

\begin{abstract}
Os estudos sobre as inter-relações endocrinológicas em bovinos da Raça Brava de Lide são raros, permanecendo ainda incompreendidos todos os mecanismos que concorrem para a sua agressividade. $A$ forma como a deficiência de testosterona ajusta exactamente o metabolismo do colesterol e da vasopressina permanece incerto. Foram estudados 10 touros da Raça Brava de Lide, dos quais, logo após o abate, foi recolhido e analisado o sangue, com o objectivo de se determinar os níveis de Testosterona, Colesterol e Vasopressina (ADH). Verifica-se uma conjunção de valores baixos de colesterol, altos de testosterona e de $\mathrm{ADH}$, coincidentes com uma maior agressividade. A correlação da $\mathrm{ADH}$ vs testosterona é positiva e significativa ao nível de 5\%. As médias encontradas para o Colesterol Total, a Testosterona Sérica e a ADH são respectivamente de - 139,4 mg/dL, 14,14 ng/dL e de 440,7 pg/ml. E, surge uma putativa correlação evidente, entre a Testosterona Sérica acima de 10,4 ng/dL e o Comportamento dos touros, qualificados com maior agressividade.
\end{abstract}

\section{Testosterone, Cholesterol, ADH and the Aggressiveness in Fighting Bulls under Stress}

\section{SUMMARY}

\section{ADDITIONAL KEYWORDS}

Hormones

Stress.

Vasopressin.

Behavior.

Bullfighting.

\section{INFORMATION}

\section{Cronología del arríiculo.}

Recibido/Received: 27.03.2019

Aceptado/Accepted: 08.07.2019

On-line: 15.10 .2019

Correspondencia a los autores/Contact e-mail:

augusto.gouveia@iniav.pt

\section{INTRODUÇÃO}

O Stress é uma resposta cumulativa que resulta da interacção do animal com o seu ambiente através de receptores (Gouveia et al. 2017). Os comportamentos expressam o resultado final de um estado fisiológico no qual podem estar envolvidas várias hormonas; a relação entre hormonas e comportamento é biunívoca (Oliveira 1994). As hormonas não causam comportamentos, mas sim alteram a probabilidade de dado comportamento acontecer num contexto social apropriado, isto é, as hormonas influenciam o Comportamento (Giaquinto S/ Data). Para que uma hormona modifique o comportamento, deve ligar-se apenas aos 
receptores com alta afinidade e especificidade para essa hormona em particular (Silver and Kriegsfeld 2016).

A divisão entre o sistema nervoso e o endócrino está a tornar-se mais indistinta à medida que compreendemos melhor como o sistema nervoso regula a comunicação hormonal (Nelson 2018).

É desconhecida a forma como a deficiência de testosterona ajusta o metabolismo do colesterol e da vasopressina (Cai et al. 2015).

São múltiplos os factores que concorrem para a agressividade despoletados pelo sistema límbico e outras estruturas neuroencefálicas e hormonais (Gouveia, Orge e Carvalho 2016)

\section{TESTOSTERONA}

Para a Neurociência, a testosterona está ligada à agressão e à violência. A testosterona também está relacionada com a vasopressina e ao colesterol, produzindo comportamentos agressivos e impulsivos (Rojas 2011).

A testosterona influi na expressão de muitos comportamentos. Os modelos animais podem ser usados para comparar os efeitos organizacionais versus os efeitos activados (Neave and O'Connor 2008).

O nível sérico de testosterona não se correlaciona com a manifestação de comportamentos agressivos em touros de lide, embora haja uma tendência da agressividade quando a concentração é maior (Cabrera 2012)

Resíduos atávicos de comportamento agressivo que prevalecem na vida animal, determinados pela testosterona, permanecem atenuados no homem e suprimidos por inibições familiares e sociais (Batrinos 2012).

O Stress influencia tanto a testosterona quanto a agressividade. Sob condições de Stress, a testosterona circulante e a agressividade podem ser correlacionadas de forma mensurável, mesmo que estas variáveis não estejam causalmente ligadas (Kedenburg 1979).

A testosterona pode exercer os seus efeitos de duas maneiras: ou em receptores de androgénios após a conversão para 5-alfa-di-hidrotestosterona ou em receptores de estrogénio após a aromatização para estradiol (Figura 1) (Simpson 2001).

Os receptores de testosterona encontram-se principalmente nalguns neurónios hipotalâmicos, onde é aromatizado em estrogénios, o que determina o aumento da agressividade (Figura 1) (Giammanco et al. 2005).

A testosterona facilita a agressão ao modular os receptores (V1) da Vasopressina (ADH) no Hipotálamo Ventrolateral - VLH. (Figura 1) (Delville, Mansour, and Ferris 1996).

\section{COLESTEROL}

As hormonas esteróides (ex. Estradiol) são sintetizadas a partir do colesterol (Figura 1), nos tecidos esteroidogénicos das adrenais, gónadas e placenta (Gonzalez e Silva 2006). A literatura sobre colesterol e agressão

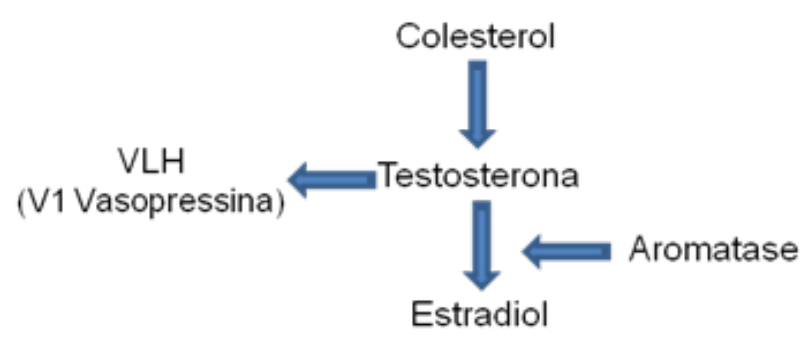

Figura 1. Modulação do Colesterol, Testosterona e Vasopressina (ADH) (Modulation of Cholesterol, Testosterone and Vasopressin $(A D H)$.

física tem consistentemente mostrado que as concentrações séricas de colesterol estão negativamente associadas à agressão física (Hillbrand and Spitz 1999).

Foi questionado se o aumento da mortalidade suicídios, homicídios e acidentes entre algumas pessoas se deveu a baixo colesterol (Jones 1998). O colesterol baixo e o comportamento violento podem estar relacionados com a diminuição da transmissão de serotonina, o que pode levar a uma pior supressão do comportamento impulsivo. Tem sido sugerido que níveis baixos de colesterol no sangue levaram os nossos ancestrais pré-históricos a caçar agressivamente para sobreviver (Wallner and Machatschke 2009). Níveis muito baixos de colesterol LDL, demonstraram estar associados a um risco aumentado de comportamento violento (Edgar et al. 2007). Evidências crescentes sugerem uma relação entre colesterol baixo e violência, agressão e hostilidade (Golomb, Stattin, and Mednick 2000). O colesterol pode desempenhar um papel como moderador da função serotoninérgica e interagir com a impulsividade. A redução dos níveis de colesterol provoca várias alterações no comportamento e os níveis de colesterol podem servir como um marcador de risco biológico para a violência (Tomson-Johanson, and and Harro 2018).

\section{VASOPRESSINA}

Vasopressina e peptídeos relacionados compreendem uma superfamília filogeneticamente antiga de sinais químicos em vertebrados e invertebrados. A vasopressina (arginina vasopressina, hormona antidiurética ou $\mathrm{ADH}$ ) é uma hormona peptídica formada no hipotálamo, transportada via axónios para a hipófise posterior, que a liberta no sangue (Guyton \& Hall 2006).

A ADH actua nos ductos coletores renais através dos receptores V2 para aumentar a permeabilidade à água (mecanismo dependente do $\mathrm{AMPc}$ ), o que leva à diminuição da formação de urina. Isto, aumenta o volume sanguíneo, o débito cardíaco e a pressão arterial (Den Ouden and Meinders 2016). Em cães que se comportaram mais agressivamente tiveram mais alta $\mathrm{ADH}$ total do que os cães mais calmos. Coletivamente, esses dados sugerem que a ADH endógena pode desempenhar papéis críticos na formação do comportamento social do cão, incluindo aspectos de agressão (MacLean 2017). Em mamíferos não humanos, o neuropeptídeo vasopressina é mediador-chave de comportamentos 
sociais complexos, incluindo a agressão. A ADH tem sido implicada principalmente em comportamentos sociais de machos, incluindo agressão (Heinrichs and Domes 2008) e, a actividade da vasopressina parece ligada ao sistema da serotonina, fornecendo um mecanismo para regular o comportamento agressivo (Ferris 2005).

Pelo antes exposto, o objectivo da investigação, abordou a inter-relação que guardam a testosterona, o colesterol e a hormona antidiurética (ADH) entre si, o que pode potenciar a agressividade sendo este, um estudo novo em animais nomeadamente em bovinos de raça brava de lide.

\section{MATERIAL E MÉTODOS}

Durante a Temporada de 2018, em Setembro foram lidados a cavalo 10 touros na Praça de Touros da Moita e no Campo Pequeno em Lisboa; na sequência foram transportados para o Matadouro Regional de Mafra, licenciado sob o Registo PTR95. Este matadouro é uma pequena Empresa Privada e foi especialmente concebido para bovinos, nomeadamente de raça brava, está devidamente e legalmente Registado na Direcção Geral de Veterinária (DGV) sob Licença de Exploração tendo que cumprir com todas as regras éticas e de bem-estar animal de acordo com as directivas internacionais emitidas por aquela Direcção Geral, obtendo os funcionários do matadouro Cursos para o efeito na DGV. O corpo inspectivo é o garante diário de vigilância e monitorização da ética e do bem-estar animal. Portanto, apesar de não ser obrigatório, as pequenas empresas privadas possuírem um código de ética, a mesma é salvaguardada pelas disposições do Estado Português que superintende nos abates sanitários.

Foram assim, considerados 10 Touros de Lide (TL), com a média de 53 meses de idade, cujo peso médio em canal a frio foi de $319,02 \mathrm{~kg}$, para um peso vivo estimado (médio) de $531,7 \mathrm{~kg}$. Após insensibilização por morte cerebral foi recolhido sangue cerca de 10 segundos depois, para 20 Eurotubos com gel de separação de soro (10) e com EDTA (10) com capacidade para 5 $\mathrm{ml}$ de sangue total. Foram transportados de imediato para o laboratório de análises - LABAMARO, sob licença da Arslvt n ${ }^{\circ}$ 00101L/2008 e com o $n^{\circ}$ de registo da ERs - E107514; Certificado pela Np EN Iso 9001 e pela EIc. O Colesterol Total foi determinado pelo Método Enzimático/Colorimétrico; a Testosterona Sérica foi determinada por Radioimunoensaio e a ADH (Vasopressina) por Quimioluminescência.

Todos os dados registados foram analisados através do software SPSS V12. O SPSS é um software aplicativo do tipo científico, cujo nome é acrónimo de "Statistical Package for Social Sciences" - Pacote este, de apoio à tomada de decisão que inclui: aplicação analítica, mineração de dados, mineração de texto e estatística que transformam os dados em informações importantes, complementado com a folha de cálculo Microsoft Office Excel 2013 com "Kaddstat: statistical analysis plug-in to Excel Microsoft Excel" (Harnett and Horrel 1998). Este programa, utiliza uma abordagem integrada e enfatiza a modelagem e a aplicação de métodos puros em vez de técnicas estatísticas. Essa ênfase permite aprender a resolver problemas, não equações matemáticas, e pre- para a tomada de decisão. Todos os modelos e análises usam o Excel sem precisar de realizar cálculos difíceis. O programa também é acompanhado pelo KaddStat, um add-in fácil de usar para o Excel, que facilita a execução de testes estatísticos complexos no Excel. Deste modo, estabeleceram-se a análise e o apuramento de dados para os TL, como médias, máximos, mínimos e o desvio padrão, bem como a elaboração de Diagramas de Extremos e Quartis (Boxplot), que, por exemplo, representam graficamente os extremos e os quartis de um conjunto de dados (o mínimo, o primeiro quartil, o segundo quartil ou mediana, o terceiro quartil e o máximo da amostra). Assim se constrói um rectângulo (caixa), do primeiro ao terceiro quartil. Uma linha vertical atravessa o retângulo na mediana. Fazemos uma linha horizontal do primeiro quartil ao mínimo e do terceiro quartil ao máximo. O Teste T-Student (Raju 2005), compara médias sendo um teste de hipóteses e constitui uma forma de inferência estatística para rejeitar ou não uma hipótese nula quando a estatística do teste $(t)$ segue uma distribuição $t$ de Student, pode ser conduzido para: comparar uma amostra com uma população, comparar duas amostras pareadas e comparar duas amostras independentes. Análise de clusters - é um procedimento estatístico multivariado que serve para identificar grupos homogéneos nos dados, com base em variáveis ou em casos. A análise de clusters, ou análise de agrupamentos, permite assim, classificar objetos e pessoas com base na observação das semelhanças e das dissemelhanças: dado um conjunto de $n$ indivíduos, sobre os quais existe informação de $p$ variáveis, o método agrupa os indivíduos em função da informação existente, de modo que os indivíduos de um grupo sejam tão semelhantes entre si quanto possível e tão diferentes dos restantes grupos quanto possível. A análise de clusters pretende organizar um conjunto de casos em grupos homogéneos, de tal modo que os indivíduos pertencentes a um grupo são o mais semelhante possível entre si e diferentes dos restantes. O dendograma (ligações médias entre grupos - "average linkage), é um tipo específico de diagrama ou representação icónica que organiza determinados factores e variáveis. Resulta de uma análise estatística de determinados dados, em que se emprega um método quantitativo que leva a agrupamentos e à sua ordenação hierárquica ascendente - o que em termos gráficos se assemelha aos ramos de uma árvore que se vão dividindo noutros sucessivamente. Isto é, ilustra o arranjo de agrupamentos derivado da aplicação de um "algoritmo de clustering".

A quantificação da amostra para a análise estatística foi determinada a partir de um cálculo simples, que pode ser usado quando a dimensão da população é conhecida, a qual refere que a dimensão de uma amostra proveniente de uma população finita, de tamanho $\mathrm{N}$ é dado por: $\mathrm{N}^{\wedge}(1 / 2)+1$ arredondado para o número inteiro mais próximo (Arsham 2015). Deste modo, a população conhecida e finita de touros de lide abatidos em setembro de 2018 no Matadouro Regional de Mafra foi de 76, de que resultou numa amostragem de 10 animais aceites.

A Associação Portuguesa de Criadores de Touros de Lide (APCTL), avaliou o Comportamento em Lide nas 
Praças de Touros onde foram corridos, numa lista que apenas continha as Identificações, à frente das quais, se classificaram os touros usando: $\mathbf{M}$ - Mau (atitudes de defesa, investidas curtas; $\mathbf{R}$ - Regular (atitudes típicas de bravo) e B - Bom (bravos com investidas permanentes). A partir da análise valorativa do comportamento em lide elaborou-se um gráfico, que permitiu demonstrar a interdependência com a Testosterona Sérica.

\section{RESULTADOS}

Os resultados obtidos nas análises efectuadas ao Colesterol Total, Testosterona Sérica e ADH, considera-

Tabela I .Valores analíticos (Analytical Values).

\begin{tabular}{cccc}
\hline & $\begin{array}{c}\text { Colesterol T } \\
\mathrm{mg} / \mathrm{dL}\end{array}$ & $\begin{array}{c}\text { Testosterona S } \\
\mathrm{ng} / \mathrm{dL}\end{array}$ & $\begin{array}{c}\mathrm{ADH} \\
\mathrm{pg} / \mathrm{mL}\end{array}$ \\
\hline Média & 139,4 & 14,14 & 440,7 \\
Máximo & 204 & 34,40 & 631,57 \\
Minimo & 113 & 3,20 & 210,33 \\
Desvio Padrão & 27,43 & 10,27 & 130,66 \\
\hline
\end{tabular}

das as médias, máximos e mínimos bem como o desvio padrão são os que se apresentam na Tabela I.

As correlações entre os dados obtidos nas análises, são demonstradas de forma relevante através das Figuras 2 a 4, cuja clareza de exposição comprova a relação dos dados obtidos.

A figura 2 mostra uma correlação ( $r=0,600916)$ e uma linha de tendência negativa, mas não significativa $(>5 \%)$.

Isto é, esta correlação inversa, de forma coadjutora, remete para um putativo e potencial, aumento da agressividade

A figura 3 mostra uma correlação $(r=0,688622)$ é positiva e significativa ao nível de $5 \%$. Fisiologicamente correcta dada a acção da testosterona em situações de stress e a $\mathrm{ADH}$, esta, enquanto vasoconstritora per-

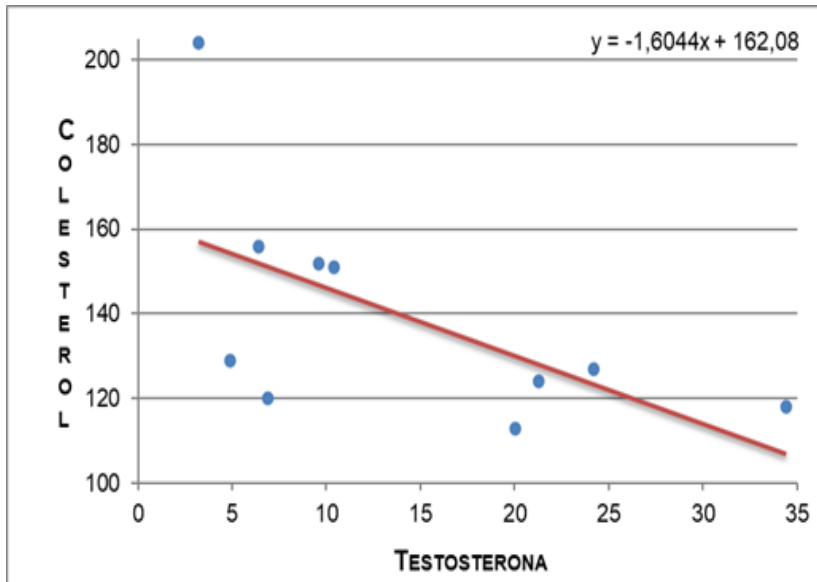

Figura 2. Correlação Colesterol Versus Testosterona (Correlation Cholesterol Versus Testosterone). mite ao Organismo potenciar a sua defesa e a fuga ou luta, aumentando a volémia

A figura 4 mostra uma correlação $(r=0,551362)$ com uma Linha de tendência negativa, mas não significativa (> 5\%).Considerada a relação significativa, directa e positiva da $\mathrm{ADH}$ e a testosterona, esta relação inversa corrobora o comportamento da ADH e do colesterol, dado que este, denota também uma diminuição face a uma subida da testosterona.

Procedeu-se à execução do teste de t para os grupos "Bom" e "Regular", assim como aos Diagramas "Whisker ou Boxplot" apresentados nas Figuras 5 a 7 que põem em evidência os valores da Tabela I de forma relevante:

O diagrama de extremos e quartis (Boxplot) para os valores do Colesterol Total mostra o posicionamento entre os valores observados e onde o teste $t$ demonstra que as médias do grupo "Bom" e "Regular" não são diferentes entre si.

O diagrama de extremos e quartis (Boxplot) para os valores da Testosterona Sérica mostra a diferença entre médias e comprova que a dispersão de valores no grupo Bom é maior que a do grupo Regular. O teste $\mathrm{t}$

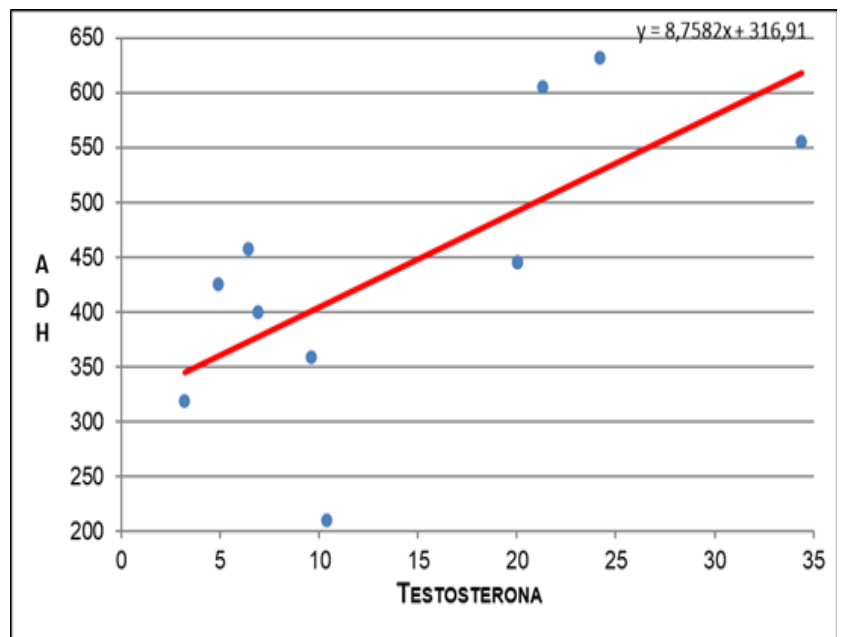

Figura 3. Correlação ADH Versus Testosterona (Correlation ADH Versus Testosterone).

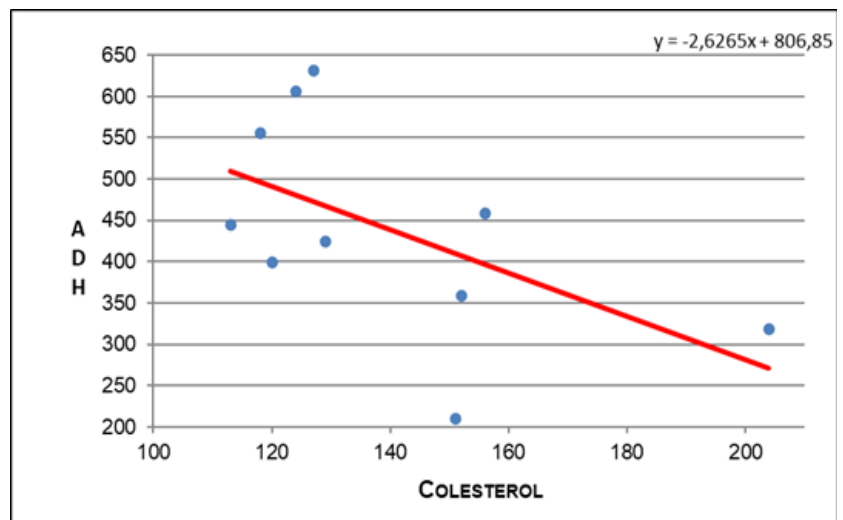

Figura 4. Correlação ADH Versus Colesterol (Correlation ADH Versus Cholesterol). 
indica que as médias do grupo "Bom" e "Regular" são diferentes entre si ao nível de $1 \%$.

Observa-se um valor extremo referente ao Touro $\mathrm{n}^{\circ}$ 1 no Comportamento Regular.

O diagrama de extremos e quartis (Boxplot) para os valores da ADH mostra o posicionamento da diferença entre médias e evidencia a dispersão de valores no grupo Bom maior que a do grupo Regular. O teste $\mathrm{t}$ indica que as médias do grupo "Bom" e "Regular" são diferentes entre si ao nível de 5\%.

Foi também realizada uma análise de grupo de que resultou um Dendograma (Figura 8) obtido por

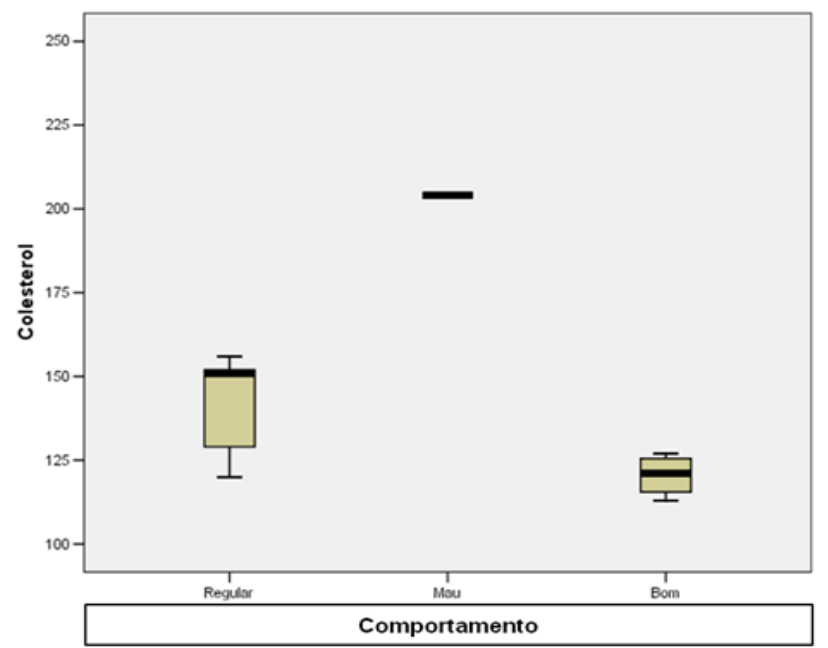

Figura 5. "Whisker ou Boxplot" mostra a distribuição dos dados em quartis e destaca as médias do Colesterol ("Whisker ou Boxplot" shows the distribution of data in quartiles and highlights the averages of Cholesterol). O Teste T-Student: Diferença Não Significativa $->0,05(>0,5 \%)$. Médias = do grupo Bom - 120,5 mg/dL; do grupo Regular - 139,25 mg/dL e, o valor do touro "Mau" - 204,00 mg/dL.

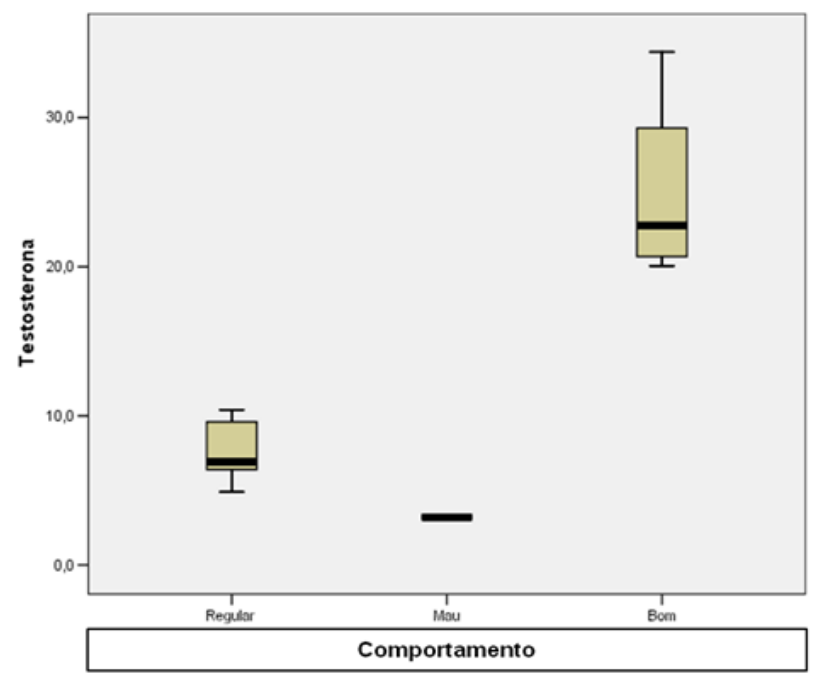

Figura 6. "Whisker ou Boxplot" mostra a distribuição dos dados em quartis e destaca as médias da Testosterona ("Whisker ou Boxplot" shows the distribution of data in quartiles and highlights the averages of Testosterone). O Teste T-Student: Diferença Muito Significativa $-0,01^{* *}(1 \%)$.Médias $=$ do grupo Bom - 24,99 ng/dL; do grupo Regular - 6,95 ng/dL e, o valor do touro "Mau" $-3,20 \mathrm{ng} / \mathrm{dL}$.

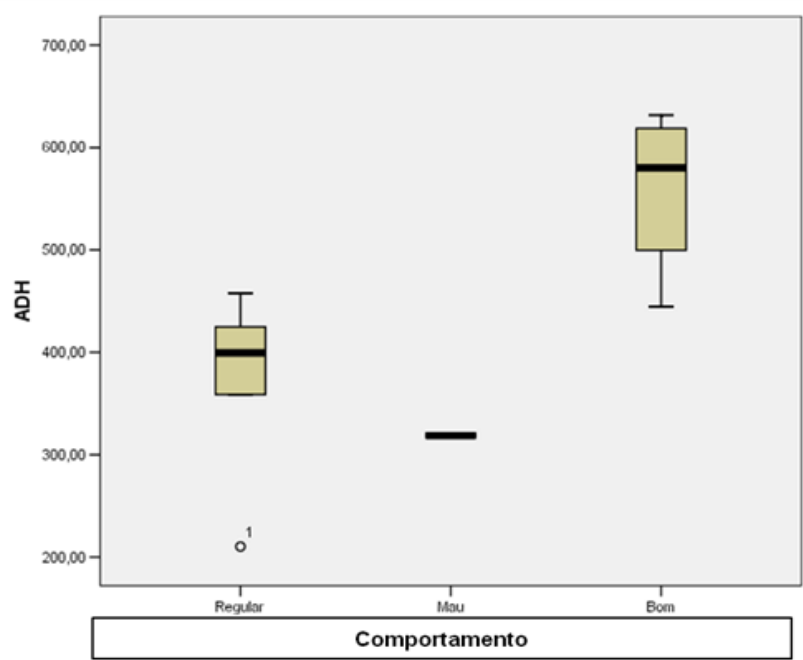

Figura 7. "Whisker ou Boxplot" mostra a distribuição dos dados em quartis e destaca a médias da ADH com um valor atípico no grupo Regular ("Whisker ou Boxplot" shows the distribution of data in quartiles and highlights the averages of $\mathrm{ADH}$ with an atypical value in the Regular group). O Teste T-Student: Diferença Significativa $-0,05^{*}(5 \%) \cdot$ Médias $=$ do grupo Bom - 559,21 pg/mL; do grupo Regular - 410,29 pg/mL e, o valor do touro "Mau" - 318,81 pg/mL.

submissão das variáveis observadas (Colesterol, Testosterona e da ADH) a análise hierárquica de "clusters" utilizando o método da ligação entre grupos e o quadrado da distância euclidiana.

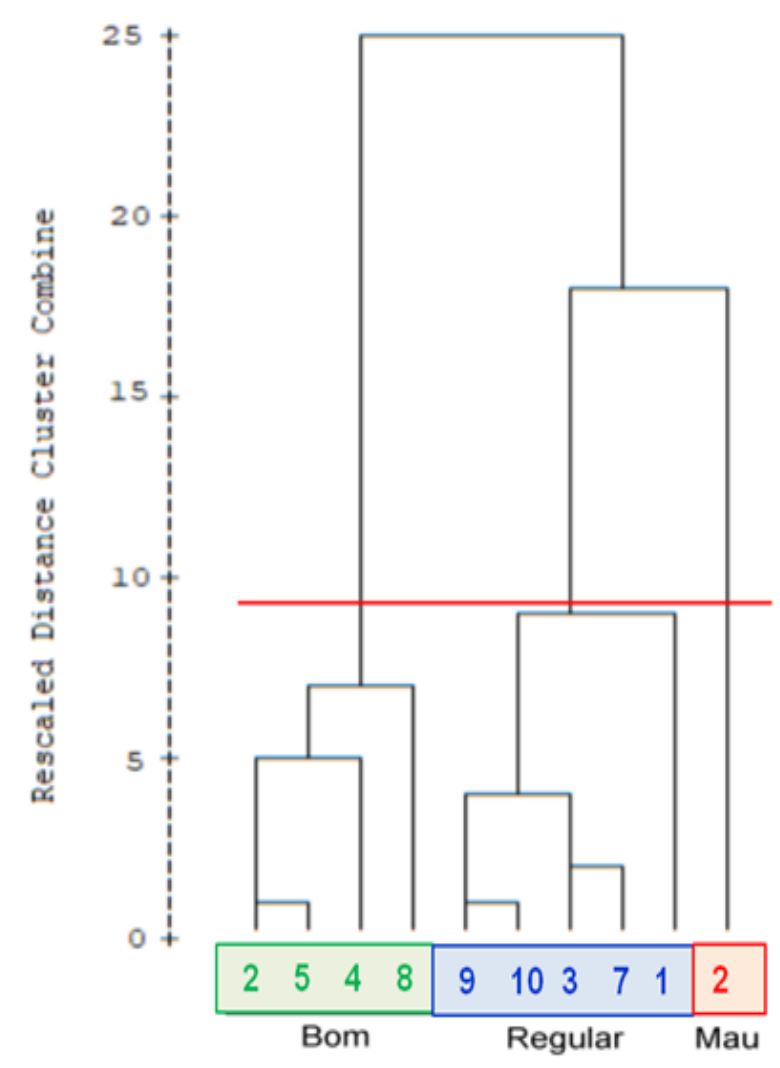

Figura 8. Dendrograma usando Ligação Média (Entre Grupos) (Dendogram using Average Linkage (Between Groups). 
Ao considerar-se uma partição de modo a lograr 3 grupos obteve-se a seguinte distribuição dos touros: $(2,5,4$ e 8$) ;(9,10,3,7$ e 1$)$; (2) a qual mostra total concordância com a classificação prévia realizada durante a "lide" de respectivamente de "Bom", "Regular" e "Mau".

- Esta distribuição dos TL por grupos apresenta seguintes características:

\section{Grupo 1.}

Touros $\mathrm{n}^{\circ}: 2,5,4$ e 8 = Este grupo com Comportamento Bom apresenta Colesterol Baixo, Testosterona e ADH Altos.

\section{Grupo 2.}

Touros $n^{\circ}$ 9, 10, 3, 7 e 1 = Este grupo com Comportamento Regular apresenta Colesterol com valor idêntico ao grupo Bom. Nos casos da Testosterona e ADH apresentam valor diferente e inferior ao grupo "Bom".

\section{Grupo 3.}

Touro $\mathrm{n}^{\circ} 2=$ Este TL com Comportamento Mau apresenta o Colesterol com o valor observado mais alto e para a Testosterona e ADH expõe, em cada caso, o valor observado mais baixo.

Solicitada à Associação Portuguesa de Criadores de Touros de Lide a valoração do comportamento em corridas de touros à Portuguesa foi-nos transmitido que 4 touros tiveram um comportamento verdadeiramente de bravos (BOM) e que 5 cumpriram, demonstrando uma atitude REGULAR na lide, foi ainda assinalado um touro com MAU desempenho apresentando características de manso. Assim, surge uma percentagem de $40 \%$ de Touros Bons, 50\% de Regulares e 10\% de Maus.

Após tratamento estatístico surgiu uma correlação evidente, demonstrada abaixo (Figura 9), entre a Testosterona Sérica acima de 10,4 ng/dL e o Comportamento de todos os Touros Bravos, qualificados de Bom.

Os Touros com Comportamento Regular apresentam valores acima de 3,2 e abaixo de 10,4 ng/dL.

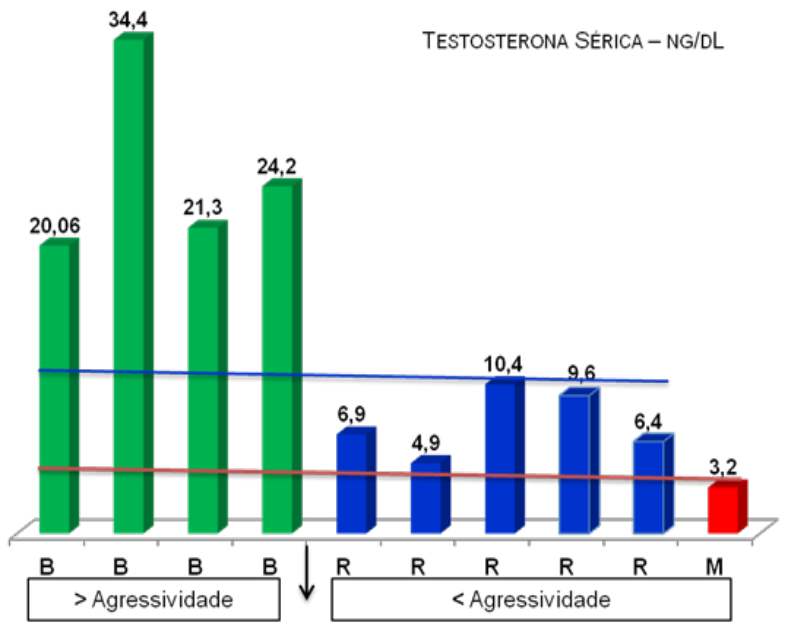

Figura 9. Correlação Significativa da Testosterona com o Comportamento (Significant Correlation of Testosterone with Behavior).
No Touro que denotou um Mau Comportamento ocorre o valor de 3,2 ng/dL.

\section{DISCUSSÃO}

As investigações em touros de lide, particularmente na área da Fisiologia são raras.

O toro de lide é um animal sui generis especificamente criado para a luta que sugere, quando isolado, comportamentos de predação apesar de ser um herbívoro ruminante.

Alguns mecanismos hormonais fisiológicos que se relacionam com a agressividade foram aqui demonstrados, que pela sua natureza é exponencial nos touros considerados mais agressivos apesar da variabilidade entre indivíduos.

Assim, verifica-se uma conjunção de valores baixos de colesterol, altos de testosterona e de ADH, coincidentes com uma maior agressividade.

Neste estudo, o tamanho da amostra é pequeno resultando em tendências e indícios. Contudo, dimanam dela, efeitos modais que podem apesar de tudo ser concludentes, isto porque, são corroborados pelos autores referenciados que elucidam o comportamento do Colesterol, Testosterona e ADH quando correlacionados com a agressividade.

Assim, referem aqueles autores que para um colesterol baixo, correspondendo a uma testosterona alta com consequente subida da $\mathrm{ADH}$, esta conjugação se pode relacionar com um aumento de agressividade.

Os dados recolhidos e analisados neste estudo demonstraram inequivocamente tal desiderato, com valores claros e objectivos podendo tirar-se ilacções, isto é, as correlações do colesterol vs testosterona e da ADH vs colesterol sendo não significativas apresentam tendências fortes e evidentes; a correlação da ADH vs testosterona é significativa e fisiologicamente correcta.

Sendo certo que a agressividade tem origem em múltiplos factores intrínsecos e extrínsecos que concorrem para a sua manifestação, é relevante que os touros após a lide a cavalo, transportados ao matadouro e esperando sossegados por mais de 6 horas o abate, em currais apropriados, $40 \%$ deles tenham ainda elevados níveis de testosterona.

Por estas razões consideramos que seria mais relevante considerar que o valor da testosterona para uma alta agressividade se poderia situar acima da média da encontrada nos touros considerados bons (+ bravos), o que significa considerar todos aqueles que possuem um nível acima de 24,99 ng/dL como os mais aptos para a lide.

Receberam os autores a valoração do comportamento dos touros em lide, da Associação Portuguesa de Criadores de Touros de Lide, através do Secretário Técnico do Livro Genealógico da Raça Brava de Lide, esta apreciação é de facto subjectiva tendo sido considerados - o "trapío", a bravura, a "casta", a acometividade, a distância da investida e a sua repetição, a força 
e a resiliência etc., etc., que no seu conjunto remeteram para a ponderação da agressividade.

Devido a esta complexidade de avaliações e marcos, que confluem para a valoração do comportamento em lide, foi decidido usar apenas o termo "agressividade" para melhor compreensão dos leigos na matéria, ainda que se mantenha a sua abrangência.

\section{CONCLUSÃO}

A correlação da ADH vs testosterona é positiva e significativa ao nível de 5\%.

As médias encontradas para o Colesterol Total, a Testosterona Sérica e a ADH são respectivamente de $139,4 \mathrm{mg} / \mathrm{dL}, 14,14 \mathrm{ng} / \mathrm{dL}$ e de 440,7 pg/ml.

Os dados encontrados para a testosterona apresentam uma Diferença Muito Significativa - 0,01** $(1 \%)$. Com as médias para o grupo Bom - 24,99 ng/dL; do grupo Regular - 6,95 ng/dL e, o valor do touro Mau 3,20 ng/dL..

Para a ADH uma Diferença Significativa - 0,05* (5\%) com médias do grupo Bom - 559,21 pg/mL; do grupo Regular - 410,29 pg/mL e, o valor do único touro $\mathrm{Mau}-318,81 \mathrm{pg} / \mathrm{mL}$.

E, para o colesterol uma Diferença Não Significativa - > 0,05 (> 0,5\%), com médias do grupo Bom - 120,5 $\mathrm{mg} / \mathrm{dL}$; do grupo Regular - 139,25 mg/dL e, o valor do único touro Mau - 204,00 mg/dL.

Surge uma putativa correlação evidente, entre a Testosterona Sérica acima de 10,4 ng/dL e o Comportamento dos touros, qualificados com > agressividade,

Os touros com Comportamento Regular apresentam valores acima de 3,2 e abaixo de 10,4 ng/dL (agressividade típica).

No touro que denotou um Mau Comportamento (< agressividade), ocorre o valor de 3,2 ng/dL.

\section{AGRADECIMENTOS}

Dr. Vasco Lucas e Associação Portuguesa de Criadores de Touros de Lide

Dr. Eurico Esteves, Administrador do Matadouro Regional de Mafra.

Labamaro - Laboratório de Análises do Alto de Santo Amaro, S.A.

Clínica de Medicina e Ortopedia Dr. Basílio de Castro, Lda.

\section{BIBLIOGRAFIA}

Arsham, H 2015, Statistical thinking for managerial decisions. In: Dr. Arsham's statistics site. Edition online by Dr. Hossein Arsham. http:// home.ubalt.edu/ntsbarsh/business-stat/opre504.htm (28/09/2018).

Batrinos, LM 2012, Testosterone and Aggressive Behavior in Man Int J Endocrinol Metab. 2012;10(3): DOI: 10.5812/ijem.3661. Pp 563-568

Cabrera, GF 2012, Variables neuroendocrinas y su relación con el comportamiento durante la lidia del toro bravo (Bos taurus, L.). [Tesis Doctoral]. Facultad Veterinaria. Universidad Complutense de Madrid. Pp. 189-211.
Cai, Z, Xi, H, Pan, Y, Jiang, X, Chen, L, Cai, Y, Zhu, K, Chen, C, Xu, X, and Chen, M 2015, Effect of testosterone deficiency on cholesterol metabolism in pigs fed a high-fat and high-cholesterol diet. Lipids Health Dis. P. 14: P 18. Mar 7. Abstract. Doi: 10.1186/s12944-015-0014-5.

Delville, Y, Mansour, KM, and Ferris CF 1996, Testosterone facilitates aggression by modulating vasopressin receptors in the hypothalamus. Physiol Behav. Jul; 60 (1): Pp 25-29.

Den Ouden, DT and Meinders, AE 2016, Vasopressin: physiology and clinical use in patients with vasodilatory shock: a review. The Netherlands Journal of Medicine 63: 2005.Revised 12/8/16. Pp 4-13,

Edgar, PF, Hooper, AJ, Poa, NR, and Burnett, JR 2007, Violent behavior associated with hypocholesterolemia due to a novel $A P O B$ gene mutation. Molecular Psychiat, 12, Pp. 258-263

Ferris, CF 2005, Vasopressin/oxytocin and aggression. Novartis Found Symp.; 268:190-8; discussion PMID: 16206881. [Indexed for Medline]. Extraído de https://www.ncbi.nlm.nih.gov/pubmed/16206881 acedido em 04.09.2018. Pp 198-200, 242-53.

Giammanco, M, Tabacchi, G, Giammanco, S, Di Majo, D, La Guardia, M 2005, Testosterone and aggressiveness. Med Sci Monit. Abstract, Apr;1 1 (4):RA136-45. Epub Mar 24.

Giaquinto, CPS/ Data, Controle Hormonal da Comunicação Animal - IB / UNESP. Apresentação PowerPoint. Pp. 1-28. Extraído de: http:// www.ibb.unesp.br/Home/Departamentos/Fisiologia/profa.Silvia/Comunicacao_controleendocrinoPercilia.pdf acedido em 30.08.2018.

Golomb, BA, Stattin, H, and Mednick, S 2000, Low cholesterol and violent crime J Psychiatr Res, 34, Pp. 301-309.

Gonzalez, FH e Silva CS 2006, Bioquímica hormonal. In: Introdução à bioquímica hormonal. 2.ed. Porto Alegre (RS): Editora da UFRGS. 2006. Cap.7, Pp. 251-312.

Gouveia, AJ, Martins, VC, Esteves, E, e Almeida, A 2017, Níveis de GABA, Serotonina, Dopamina, Adrenalina e Noradrenalina em Touros de Lide e Bovinos Produtores de Carne sob Stress. Rev. electrón. vet. Volumen $18 \mathrm{~N}^{\circ}$ 8. ISSN 1695-7504. Pp. 1-13.

Gouveia, AJ, Orge, L e Carvalho, P 2016. A dimensão da amígdala cerebral e a agressividade no touro de lide. Arch. Zootec. 65 (249): 59-65

Guyłon, AC \& Hall, JE 2006, Textbook of Medical Physiology. $11^{\text {th }}$ Ed. Pp.602,603,635. ISBN 0-7216-0240-1. Elsevier Inc. 1600 John F. Kennedy Blvd., Suite 1800. Philadelphia, Pennsylvania 19103-2899.

Harnett, DL and Horrel, JF 1998, Statistical analysis plug-in to Microsoft Excel Version 5.0. Kaddstat Multimedia CD. Wiley. New York. USA.

Heinrichs, M and Domes, G 2008, Neuropeptides and social behaviour: effects of oxytocin and vasopressin in humans. Prog Brain Res. Abstract, 170: 337-50. doi: 10.1016/S0079-6123(08)004287. Extraído de: https://www.ncbi.nlm.nih.gov/pubmed/18655894 acedido em 04.09.2018

Hillbrand, M and Spitz, RT 1999, Aggression and Violent Behavior, Vol. 4, No. 3, Copyright Ó 1999 Elsevier Science Ltd. PII S13591789(98)00009-3. Pp. 359-370.

Jones, A, Johnson, ZC, Robinson, JD, and Ybarra, MA 1998, Cholesterol and Aggression: An Ethnocultural Perspective. Journal of Clinical Psychology in Medical Settings, Vol. 5, No. 3. Pp 249-258.

Kedenburg, D 1979, Testosterone and human aggressiveness: an analysis. Journal of Human Evolution. Volume 8, Issue 3, March, https://doi.org/10.1016/0047-2484(79)90064-2. Pp. 407-410.

MacLean, EL, Gesquiere, LR, Gruen, ME, Sherman, BL, Martin, LW, and Carter, CS 2017, Endogenous Oxyłocin, Vasopressin, and Aggression in Domestic Dogs. Frontiers in Psychology | www.frontiersin.org. doi: 10.3389/fpsyg.2017.01613. Pp 1-12.

Neave, N and O'Connor, D 2008, Testosterone and male behaviours. .Hormones and behaviour: A psychological approach. Cambridge: Cambridge University Press. Vol 22 no 1, Pp 28-31.

Nelson, RJ 2018, Hormones \& behavior. In R. Biswas-Diener \& E. Diener (Eds), Noba textbook series: Psychology. Champaign, IL: DEF publishers. DOI:nobaproject.com. Extraído de: https://nobaproject.com/ modules/hormones-behavior acedido em 05.10.2018 
Oliveira, RF 1994, Hormonas e comportamento - Aspectos actuais da endocrinologia comportamental. In V. C. Almada \& R. F. Oliveira (Eds.), Actas do I congresso nacional de etologia. Lisboa: ISPA. http:// hdl.handle.net/10400.12/1555 Pp. 73-82.

Raju, TN 2005, William Sealy Gosset and William A. Silverman: two students of science. Pediatrics, 116: Pp 732-735.

Rojas, A 2011 , Hormonas están involucradas en la violencia. Fundación Universitaria San Martin. Extraído de: http://medicinafusmviolencia. blogspot.com/2011/10/que-hormonas-estan-involucradas-en-la.html acedido em 12.09.2018.
Silver, R and Kriegsfeld, JL 2016, Hormones and Behaviour. Encyclopedia of Life Sciences (), John Wiley \& Sons, Ltd. DOI: 10.1038/npg. els.0000146 www.els.net. Pp 1-10.

Simpson, K 2001, The Role of Testosterone in Aggression. Mcgill J Med 2001 6: Pp 32-40.

Tomson-Johanson, K and Harro, J 2018. Low cholesterol, impulsivity and violence. Endocrinology \& Diabetes and Obesity: April 2018 - Volume 25 - Issue 2 - Pp 103-107. Doi: 10.1097/MED.0000000000000395. LIPIDS: Edited by Dan Streja.

Wallner, B and Machatschke, $1 \mathrm{H} 2009$, The evolution of violence in men: the function of central cholesterol and serotonin Prog Neuropsychopharmacol Biol Psychiat, 33, Pp. 391-397 